\title{
Telehealth approaches to suicide prevention: a review of evidences
}

\author{
Dr. R.B. Marasinghe \\ Department of Medical Education, University of Sri Jayewardenepura, Sri Lanka
}

\section{Dr. D. Kavanagh}

Institute of Health \& Biomedical Innovation, Queensland University of Technology, Australia

\section{Dr. S. Edirippulige}

Centre for Online Health, University of Queensland, Australia.

\section{Dr. A. Smith}

Centre for Online Health, University of Queensland, Australia

\section{Prof. M.T.M. Jiffry}

Department of Medical Education, University of Sri Jayewardenepura, Sri Lanka

Contact e-mail address: rohanabm@yahoo.com

eHealth Sri Lanka 2010,1(suppl.1):S8

DOI: http://dx.doi.org/10.4038/sljbmi.v1i0.3540

Only the Abstract is available

\begin{abstract}
Introduction: Suicide is a major preventable global problem. In Sri Lanka, suicide rate is double the world's average but healthcare resources are limited. Use of Information and Communication Technologies (ICT) to deliver psychological care over distance, tele-psychiatry is a promising alternative. However, evidence is needed to justify the wider application of tele-health in suicide prevention.

Objective: Main objective is to review published evidence of tele-health approaches to suicide prevention.

Method: The electronic databases Ovid Medline and PubMed were accessed in 2009. The appropriate search terms for Ovid Medline and MeSH terms for PubMed database search was used. Finally, the selected articles were reviewed closely. Results: Results were categorised into Randomised Clinical Trial (RCT) and non RCT evidences. In RCT trials, the tele-health approaches were superior when Cognitive therapy (CT) was incorporated. However, this superior effect was not observed when Manual-assisted cognitive therapy (MABCT) was compared with TAU. Similarly, no significant reduction was seen when Dialectical Behaviour Therapy (DBT) was compared with TAU. However, DBT and transference-focused psychotherapy were superior to TAU over time. Tele-health applications were also effective when Problem Solving (PS) component was incorporated. A significantly superior effect found with the interpersonal problem-solving (IPS) than TAU. However, task centred case work (applying Problemsolving therapy) and TAU have shown similar effects. The Interpersonal Problem-Solving Skills Training (IPSST) had not added advantage over brief problem-oriented approach. Moreover, family problem-solving sessions were not found to be superior to TAU. In contrast, non RCT evidences were vary and Real-time and Store-and-forward applications were equally used at various levels (ie. pre-intervention, intervention, post-intervention and education and training).

Conclusion: Tele-health approaches to suicide prevention are effective when underline psychological method (Cognitive Behaviour Therapy and Problem Solving Therapy) was incorporated. Notably, the telephony has been used in most tele-health suicide prevention approaches.
\end{abstract}

Keywords - tele-health, suicide prevention, PubMed database, problem-solving sessions 
